\title{
Would Janus' view on HDL be useful?
}

\author{
Kasko M, Kasko V, Oravec S \\ 1st Department of Internal Medicine, Faculty of Medicine, Comenius University, Bratislava, Slovakia. \\ kasko.martin@gmail.com
}

\begin{abstract}
Low-density lipoprotein cholesterol (LDL-C) is generally considered to be pro-atherogenic and high-density lipoprotein cholesterol (HDL-C) to be anti-atherogenic. The clinical approach to the diagnostics and treatment of clinical manifestations of atherosclerosis is the examination of the lipid spectrum. In routine clinical practice, the effects of the HDL class are measured only by determining the concentration of HDL cholesterol. It is questionable whether this clinical approach provides sufficient information to evaluate both the overall cardiovascular risk and the effect of hypolipidemic therapy. Recent studies revealed a shift from large to small HDL particles within the HDL family in the state of atherosclerotic cardiovascular diseases (CVD). This trend of lipoprotein constellation seems to be pathognomonic for dysfunctional lipoprotein profile under pathological state of the cardiovascular system. Thus, the diagnostic and therapeutic approach based on "good" cholesterol concept needs remedying (Fig. 1, Ref. 28). Text in PDF www.elis.sk.
\end{abstract}

KEY WORDS: HDL, cholesterol, lipoproteins, atherosclerosis.

\section{Introduction}

Cardiovascular (CV) system homeostasis is substantially dependent on lipid transport to the tissues provided by lipoproteins. The disturbed interaction between lipoproteins and the vascular wall - especially endothelium, induces atherosclerotic changes. Atherosclerosis as the leading cause of CVD is the crucial cause of mortality in Europe, with $45 \%$ of all deaths (accounting for 4 million) and with 1.4 million of these deaths being premature. Coronary heart disease (CHD) represents the major cause of CVD death, with $20 \%$ of all deaths (1). Based on clinical and epidemiological studies, it is well known that levels of blood lipids/lipoproteins are closely and independently associated with CVD; low-density lipoprotein (LDL) cholesterol directly and high-density lipoprotein (HDL) cholesterol inversely (2). After long-standing substantial body of evidence HDL cholesterol has started to be labelled as the "good" cholesterol, especially for its ability to dispose cholesterol from blood vessels. Practically the sole surrogate used in clinical praxis for examination of the whole HDL fraction (which encompasses wide lipoprotein spectrum in blood) is the concentration of HDL-C. Most recent evidence reveals controversy in the traditional concept of the "good" HDL cholesterol in the meaning of its ambivalence $(3,4,5)$. Even the more distant studies pointed out the importance of HDL subpopulation analysis and have begun to dispute the proclaimed holistic HDL protectiveness (6).

1st Department of Internal Medicine, Faculty of Medicine, Comenius University, Bratislava, Slovakia

Address for correspondence: S. Oravec, MD, PhD, 1st Department of Internal Medicine, Faculty of Medicine, Comenius University, University Hospital Bratislava, Mickiewiczova 13, SK-813 69 Bratislava, Slovakia.
Moreover, the latest US Dietary guidelines - Scientific Report of the 2015 Dietary Guidelines Advisory Committee (7) - bring lighting outcome in relationship with dietary cholesterol. There is no more restriction in cholesterol intake and dietary cholesterol is no more "risk". Guideline authorities stressed that it is the quality (composition) which plays the key role in the fat/cholesterol intake. Thus, a novel marker for more effective estimation of atherosclerotic CV risk is needed.

\section{HDL cholesterol versus HDL particles}

HDL is composed of heterogeneous circulating complexes of (apo)proteins and lipids. These high-density particles contain cholesterol, which represents the main component of the particle and can be chemically measured. On the other hand, HDL-C comprises heterogeneous lipoprotein particles with different size and compositions.

Given that under pathological condition - atherosclerosis, there is observed a shift from larger to smaller HDL particles in the HDL profile $(8,9,3,5)$, this finding supports a hypothesis that the existence of higher amount of smaller HDL particles in atherosclerotic CVD patients is due to the disturbed constellation of the HDL spectrum as a consequence of impaired function of small HDL particles. As an example can be described one mechanism under proinflammatory state when serum amyloid A (SAA), an acute phase protein, interacts with the HDL subpopulation of small particles; circulating SAA substitutes Apo A-I, the main protein component of HDL-mediated reverse cholesterol transport, and incorporated into the lipoprotein membrane produces dysfunctional SAA-rich HDL, with diminished cholesterol efflux capacity. HDL has an anti-inflammatory capability, but under chronic proinflammatory state (obesity, smoking or diabetes) HDL particles are 
245-248

trapping and inactivating the constantly generated free radicals in blood circulation (10). By overaccumulation of these substances the whole HDL population is probably becoming dysfunctional what is mirroring in the growth of small HDL levels.

Moreover, there is observed a novel mechanism of interaction between HDL particles and inflammation which involves circulating microparticles (MPs) and microRNAs (miRNAs). A schematic model „HDL on the background of influence of MPs and miRNAs" is represented by Figure $1(11-14)$. As a result of these processes the (dys)functionality of HDL projected into the size of HDL particles can be crucial for the pathophysiological basis of atherogenic dyslipidaemia and cholesterol homeostasis.

\section{HDL cholesterol and CV risk}

Current guidelines of the European Society of Cardiology/ European Association of Cardiovascular Prevention and Rehabilitation (ESC/EACPR) concerning CVD prevention, recommend improvement of blood cholesterol by targeting LDL-C, but not HDL-C. However, achieving the goals for LDL-C even with intensive statin therapy could not prevent many of CV events (15). Measuring the concentration of HDL-C is in daily clinical praxis usually used to evaluate the effect of HDL family/spectrum.

The main roles of high-density lipoprotein fraction are:

- reverse cholesterol transport - CETP (cholesteryl ester transfer protein) - from the periphery back to the liver,

- a source of cholesterol for biostructures,

- the esterification of free cholesterol by LCAT (lecithin-cholesterol acyltransferase).

HDL exhibits a variety of properties that act against atherogenesis (16):

- endothelial function enhancement,

- inhibition of formation/effect and incorporation of the oxidized LDL,

- suppression of proinflammatory processes,

- immunomodulatory effects,

- thrombomodulatory function, and is an important modifier when total CV risk (SCORE system) is calculated. Extended view on these relationship leads to step-by-step uncovering of residual risk as a serious obstacle in the way to further reduction of atherosclerotic impairment in the adult population. Even more, awareness of pre-existence of pro-atherogenic state in clinically healthy subjects (atherogenic lipoprotein profile phenotype B) as a dysfunctional composition of lipoproteins is more than challenging (17-19).

The relationship between the HDL family and atherosclerotic disease seems to be more complex as usually is accepted in clinical praxis. The Framingham study disputed already in 1977 the predictive value of the relationship between HDL cholesterol and the risk of CHD. It was for the first time when a large study identified a wide diversity of lipoprotein populations (20). It may be assumed that low HDL is a part of residual risk. On the other hand, the latest evidence showed that the current HDL-C modifying approaches (concentration increase) did not reduce CVD mortality despite observational studies suggested they did. A finding that

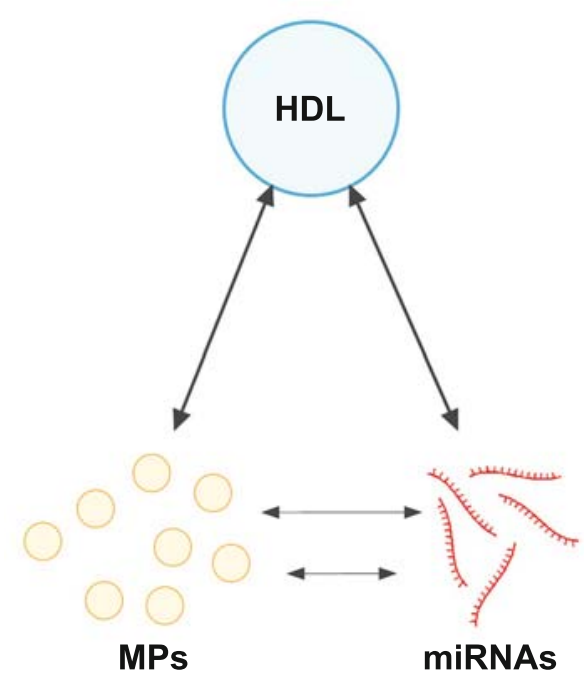

Fig. 1. HDL particle modification on the background of MPs (circulating microparticles) and miRNAs (microRNAs) interaction (schematic). MPs as extracellular microvesicles and miRNAs as a non-coding post-transcriptional regulators of gene expression may influence the functionality of HDL.

HDL-C is not always associated with CV events is emerging, and even, under type 2 diabetes condition a higher HDL cholesterol level at baseline was associated with a higher risk of CV events and all-cause mortality $(21,22)$. The impaired HDL population on the background of atherosclerosis seems to play an important role in residual risk. The role of HDL in the pathogenesis of CVD arises when it comes to disturbance in the complex of structuralfunctional relationship inside the HDL fraction; potentiating endothelial dysfunction and consecutive residual risk.

\section{Clinical approach to HDL cholesterol}

The latest evidence did not confirm decrease in CVD risk by HDL cholesterol level-improving interventions in spite of existence of the independent negative correlation between HDL-C level and prevalence of CVD (23). Thus, the CVD causality for HDL-C was not proven. On the other hand, there are already available data, that inhibition of inflammation, without affecting cholesterol levels, improve CV outcome by atheroprotection (24). Anti-inflammatory therapy with canakinumab showed similar effect to monoclonal antibodies targeting proprotein convertase subtilisin-kexin type 9 on prevention of cardiovascular events.

Recent studies with high-density lipoproteins and their subfractions in relation to atherosclerotic CVD aiming the shift of understanding the "good" cholesterol as HDL cholesterol concentration to HDL properties and another component than contained cholesterol $(25,3,5,8,9)$. The subfraction-analysis diagnostic method allows examination of the $\mathrm{CV}$ risk beyond the common blood cholesterol testing. Separation of HDL subpopulations under pathological state has revealed a characteristic shift toward small HDL particles $(5,9,25)$. Significantly higher amount of 
small HDL particles and their disturbed correlation within HDL fraction supports the concept of dysfunctional lipoproteins and their subfractions.

The analysis of HDL subpopulations provides more comprehensive insight into the conventional measuring and interpretation of HDL cholesterol as an independent risk variable. In the last few years, much more information on the role of HDL has become available and an increasing number of studies have found direct association between small HDL and atherosclerotic impairment. Nowadays we are on a crossroad where it is inevitable to reconsider the traditional role of lipoproteins in the concept of atherosclerosis. It is becoming known that not just the amount of the lipid fraction, but its composition is that what matters.

The estimation of HDL subfractions can give the clinician further information about CVD risk state and thus better calculate global CV risk. Future studies on the current topic are therefore required in order to elucidate ways how to influence the highdensity lipoprotein composition rather than to raise the HDL-C spectrum en block. Whether the small HDL particles are markers of atherogenesis or play a direct role in atherogenic process a further research will clarify.

The lipoprotein subpopulation-testing provides advantageous HDL analysis in the setting of (sub)clinical atherosclerotic impairment and thus can bring actual contribution to routine clinical praxis. The clinical use of advanced lipoprotein subfraction capabilities carries additional benefits in blood cholesterol analysis and subsequent atherosclerotic CVD risk prediction with tailored primary and secondary interventions.

Reduced levels of HDL-C are usually of secondary origin, caused by smoking, abdominal obesity (as a consequence of energy dysbalance), hypertriglyceridemia and insulin resistance. Smoking cessation, regular exercise, body weight control and healthy diet improve both HDL cholesterol levels and HDL constellation with positive effect on CV outcomes $(26-28,7)$. HDL-C-neutral diet contains $\omega-3$ polyunsaturated fatty acids, monounsaturated fatty acids, saturated fatty acids. HDL-C is lowered by trans-forms of fatty acids (partially hydrogenated vegetable oils), refined carbohydrates and by $\omega-6$ polyunsaturated fatty acids.

\section{Conclusion}

The concept of dysfunctional HDL which is linked with lipoprotein particle size underlines the importance of a new approach in dyslipidaemia diagnosis and treatment: the analysis of HDL subfractions appear to be a better predictor of atherosclerotic burden and CVD. Non-pharmacological intervention to improve the HDL population, such as life style and risk factor modification, is a reasonable strategy in the paradigm of a CVD continuum. The direction of lipoprotein constellation (increasing of the concentration of small HDL particles) seems to be pathognomonic for dysfunctional lipoprotein profile under pathological state, when HDL cholesterol is generated by the number of HDL particles instead of the HDL particles size and cholesterol mass.

\section{References}

1. Townsend N, Wilson L, Bhatnagar P, Wickramasinghe K, Ravner M, Nicholas M. Cardiovascular disease in Europe - epidemiological update 2016. Eur Heart J 2016; 37 (42): 3232-3245.

2. Gordon T, Castelli WP, Hjortland MC, Kannel WB, Dawber TR. High density lipoprotein as a protective factor against coronary heart disease. The Framingham Study. Am J Med 1977; 62: 707-714.

3. Pascot A, Lemieux I, Prud'homme D et al. Reduced HDL particle size as an additional feature of the atherogenic dyslipidemia of abdominal obesity. J Lipid Res 2001; 42 (12): 2007-2014.

4. Musunuru K, Orho-Melander M, Caulfield MP et al. Ion mobility analysis of lipoprotein subfractions identifies three independent axes of cardiovascular risk. Arterioscler Thromb Vasc Biol 2009; 29: 1975-1980.

5. Oravec S, Dostal E, Dukat A, Gavornik P, Kucera M, Gruber K. HDL subfractions analysis: A new laboratory diagnostic assay for patients with cardiovascular diseases and dyslipoproteinemia. Neuroendocrinol Lett 2011; 32 (4): 502-509.

6. Johansson J, Carlson LA, Landou C, Hamsten A. High density lipoproteins and coronary atherosclerosis. A strong inverse relation with the largest particles is confined to normotriglyceridemic patients. Arterioscler Thromb 1991; 11 (1): 174-182.

7. Scientific Report of the 2015 Dietary Guidelines Advisory Committee. Washington DC: 2015 Dietary Guidelines Advisory Committee, 2015. https://health.gov/dietaryguidelines/2015-scientific-report/pdfs/scientificreport-of-the-2015-dietary-guidelines-advisory-committee.pdf

8. Goliasch G, Oravec S, Blessberger $\mathbf{H}$ et al. Relative importance of different lipid risk factors for the development of myocardial infarction at a very young age ( $\leq 40$ years of age). Eur J Clin Invest 2012; 42 (6): 631-636.

9. Kasko M, Gaspar L, Dukat A, Gavornik P, Oravec S. High-density lipoprotein profile in newly-diagnosed lower extremity artery disease in Slovak population without diabetes mellitus. Neuro Endocrinol Lett 2014; 35 (6): 531-535.

10. Hulín I, Štvrtinová V, Ferenčík M, Kiňová S. Zápal a systémové reakcie. [Inflammation and systemic reaction]. 22-25. In: Kiňová S, Hulín I (Eds). Interná medicína. [Internal medicine]. Bratislava: ProLitera, 2013.

11. Enkhmaa B, Anuurad E, Berglund L. The unresolved mystery of high-density lipoprotein: time for a paradigm shift? Transl Res 2016; 173: $1-6$.

12. Wang S, Peng DQ, Yi Y. The unsolved mystery of apoA-I recycling in adipocyte. Lipids Health Dis 2016; 15: 35.

13. Dukát A, Šimko F. Circulating microparticles - new risk factor, or biomarker for cardiovascular diseases? Cardiology Lett 2016; 25 (2): $85-90$.

14. Valášková Z, Mladosievičová B, Maruščáková L, Hulín I. MicroRNA - Information about myocardial damage or biomarker of heart failure? Cardiology Lett 2017; 26 (5): 299-302.

15. Piepoli MF, Hoes AW, Agewall S et al. 2016 European Guidelines on cardiovascular disease prevention in clinical practice: The Sixth Joint Task Force of the European Society of Cardiology and Other Societies on Cardiovascular Disease Prevention in Clinical Practice (constituted by representatives of 10 societies and by invited experts): Developed with the special contribution of the European Association for Cardiovascular Prevention \& Rehabilitation (EACPR). Eur Heart J 2016; 37 (29): 2315-2381. 
245-248

16. Oravec S, Demuth K, Myara I, Hornych A, Balažovjech I. Aterogénne a antiaterogénne lipoproteíny plazmy modulujú sekréciu prostanoidov endotelovou bunkou in vitro. [Atherogenic and antiatherogenic plasma lipoproteins are able to moderate endothelial cell prostanoid secretion in vitro.] Bratisl Lek Listy 1998; 99 (10): 525-530.

17. Oravec S, Gruber K, Dukat A, Gavornik P, Gaspar L, Dostal E. The atherogenic profile-predominant lipoprotein profile in cardiovascular disease. Atherosclerosis 2016; 252: e67.

18. Oravec S, Gruber K, Dostal E, Mikl J. Hyper-betalipoproteinemia LDL 1,2: a newly identified nonatherogenic hypercholesterolemia in a group of hypercholesterolemic subjects. Neuroendocrinol Lett 2011; 32 (3): 322-327.

19. Oravec S, Dukat A, Gavornik $P$ et al. Atherogenic versus non-atherogenic lipoprotein profiles in healthy individuals. Is there a need to change our approach to diagnosing dyslipidemia? Curr Med Chem 2014; 21 (25): 2892-901.

20. Castelli WP, Doyle JT, Gordon T, Hames CG, Hjortland MC, Hulley SB. HDL cholesterol and other lipids in coronary heart disease. The cooperative lipoprotein phenotyping study. Circulation 1977; 55 (5): 767-772.

21. van de Woestijne AP, van der Graaf Y, Liem AH, Cramer MJ, Westerink J, Visseren FL. Low high-density lipoprotein cholesterol is not a risk factor for recurrent vascular events in patients with vascular disease on intensive lipid-lowering medication. J Am Coll Cardiol 2013; 62 (20): 1834-1841.

22. Sharif S, van der Graaf Y, Nathoe HM, de Valk HW, Visseren FL, Westerink J; SMART Study Group. HDL Cholesterol as a Residual Risk Factor for Vascular Events and All-Cause Mortality in Patients with Type 2 Diabetes. Diabetes Care 2016; 39 (8): 1424-1430.
23. Keene D, Price C, Shun-Shin MJ, Francis DP. Effect on cardiovascular risk of high density lipoprotein targeted drug treatments niacin, fibrates, and CETP inhibitors: meta-analysis of randomised controlled trials including 117,411 patients. BMJ 2014; 349: g4379.

24. Ridker PM, Everett BM, Thuren T et al. Antiinflammatory Therapy with Canakinumab for Atherosclerotic Disease. N Engl J Med 2017; 377 (12): 1119-1131.

25. Krychtiuk KA, Kastl SP, Pfaffenberger $\mathbf{S}$ et al. Small high-density lipoprotein is associated with monocyte subsets in stable coronary artery disease. Atherosclerosis 2014; 237 (2): 589-596.

26. Gepner AD, Piper ME, Johnson HM, Fiore MC, Baker TB, Stein JH. Effects of smoking and smoking cessation on lipids and lipoproteins: outcomes from a randomized clinical trial. Am Heart J 2011; 161 (1): $145-151$.

27. Kraus WE, Houmard JA, Duscha BD et al. Effects of the amount and intensity of exercise on plasma lipoproteins. N Engl J Med 2002; 347 (19): 1483-1492.

28. Halverstadt A, Phares DA, Wilund KR, Goldberg AP, Hagberg JM. Endurance exercise training raises high-density lipoprotein cholesterol and lowers small low-density lipoprotein and very low-density lipoprotein independent of body fat phenotypes in older men and women. Metabolism 2007; 56 (4): 444-450.

Received December 18, 2017. Accepted January 22, 2018. 